\title{
Motion training on a validated mechanical ERCP simulator improves novice endoscopist performance of selective cannulation: a multicenter trial $\square$
}

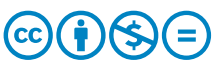

Authors

Theodor Voiosu1,2, Claudia Puscasu1 ${ }^{1}$, Beatrice Orlandini ${ }^{3}$, Masa Cavlina ${ }^{4}$, Noor Bekkali ${ }^{5}$, Leonardo Henry Eusebi ${ }^{6}$, Margherita Pizzicannella7, Daniel Blero ${ }^{8}$, Paul Balanescu ${ }^{2,9}$, Andrei Voiosu ${ }^{1}$, Silvana Perretta7 ${ }^{7}$ Nadan Rustemovic ${ }^{4}$, Lorenzo Fuccio $^{6}$, Radu Bogdan Mateescu ${ }^{1,2}$, Cesare Hassan ${ }^{10}$, Sachin Wani11, Guido Costamagna ${ }^{3,12}$, Ivo Boskoski ${ }^{3,12}$

Institutions

1 Gastroenterology Department, Colentina Clinical, Hospital, Bucharest, Romania

2 Internal Medicine Department, Carol Davila School of Medicine, Bucharest, Romania

3 Fondazione Policlinico Universitario Agostino Gemelli, IRCCS, Rome, Italy

4 Department of Gastroenterology and hepatology University Hospital Centre, Zagreb, Croatia

5 Oxford University Hospitals NHS Foundation Trust, Oxford, UK

6 Gastroenterology Unit, DIMEC, S. Orsola-Malpighi Hospital, Bologna, Italy

7 Department of Surgery, IRCAD, Strasbourg, France

8 Department of Gastroenterology, Hepatopancreatology and Digestive Oncology, Erasme Hospital, Brussels, Belgium

9 Clinical Immunology Department, Colentina Clinical Hospital, Bucharest, Romania

10 Endoscopy Unit, Nuovo Regina Margherita Hospital, Rome, Italy

11 University of Colorado Anschutz Medical Campus, Aurora, Colorado, United States

12 Centre for Endoscopic Research Therapeutics and Training (CERTT), Università Cattolica del Sacro Cuore, Rome, Italy

Bibliography

Endoscopy International Open 2021; 09: E145-E151

DOI 10.1055/a-1315-1994

ISSN 2364-3722

(C) 2021. The Author(s).

This is an open access article published by Thieme under the terms of the Creative Commons Attribution-NonDerivative-NonCommercial License, permitting copying and reproduction so long as the original work is given appropriate credit. Contents may not be used for commecial purposes, or adapted, remixed, transformed or built upon. (https://creativecommons.org/licenses/by-nc-nd/4.0/)

Georg Thieme Verlag KG, Rüdigerstraße 14,

70469 Stuttgart, Germany
Corresponding author

Claudia Puscasu, MD, Gastroenterology Department, Colentina Clinical Hospital, 19-21 Stefan cel Mare Blvd, Bucharest, Romania

Fax: +40213180604

puscasu_ic@yahoo.com

\# Supplementary material is available under

https://doi.org/10.1055/a-1315-1994

\section{ABSTRACT}

Background and study aims Current data show that traditional training methods in endoscopic retrograde cholangiopancreatography (ERCP) fall short of producing competent trainees. We aimed to evaluate whether a novel approach to simulator-based training might improve the learning curve for novice endoscopists training in ERCP.

Methods We conducted a multicenter, randomized controlled trial using a validated mechanical simulator (the Boškoski-Costamagna trainer). Trainees with no experience in ERCP received either standard cannulation training or motion training before undergoing standard cannulation training on the mechanical simulator. Trainees were timed and graded on their performance in selective cannulation of four different papilla configurations.

Results Thirty-six trainees (16 in the motion training group, 20 in the standard group) performed 720 timed attempts at cannulating the bile duct on the simulator. Successful cannulation was achieved in 698 of 720 attempts (96.9\%), with no significant difference between the two study groups $(P=0.37)$. Trainees in the motion training group had significantly lower median cannulation times compared to the standard group ( 36 vs. 48 seconds, $P=$ 0.001 ) and better technical performance on the first papilla type $(P=0.013)$.

Conclusions Our findings suggest that motion training could be an innovative method aimed at accelerating the learning curve of novice trainees in the early phase of their training. Future studies are needed to establish its role in ERCP training programs. 


\section{Introduction}

Endoscopic retrograde cholangiopancreatography (ERCP) is one of the most advanced and technically demanding therapeutic procedures in endoscopy, requiring extensive hands-on training before achieving competency and undertaking independent practice.

In most training centers threshold numbers are still used as a surrogate for competency, although there is a growing body of evidence showing that mere caseload does not guarantee competency in ERCP, with many trainees failing to reach predefined thresholds at the end of their training [1]. Current data support a paradigm shift, from the traditional volume-based approach to a competency-based approach to training in ERCP [2].

In addition to the change underway in hands-on training, simulator-based training using mechanical, digital or animal models has been proposed as a solution to shorten the learning curve in endoscopy [3-5]. While several models for ERCP have been described [6,7], the Boškoski-Costamagna mechanical trainer (BCMT) (Cook Medical) is, to our knowledge, the first mechanical model that has been independently validated and which was deemed to show good face validity as a training model for novice endoscopists in an evidence-based fashion [8].

A novel application of this model [9], which was shown to be capable of fulfilling innovative tasks such as writing or drawing with the aid of a duodenoscope and a modified ERCP catheter, was adopted by our group as the basis for motion-training. Thinking outside the box, we hypothesized that trainees unfamiliar with the handling of a side-viewing scope and accessories, could better acquire basic skills in ERCP (i.e. positioning in front of the papilla, scope and accessory handling, selective cannulation etc.) by first practicing more familiar motor tasks such as writing their names or drawing simple shapes using the BCMT and a modified ERCP catheter.

In our current study, we aimed to assess whether motion training offers an additional benefit to standard training on the BCMT in achieving the skills required for successful cannulation.

\section{Methods}

We conducted a multicenter, randomized clinical trial of trainee performance on the mechanical simulator, comparing the cannulation skills of trainees receiving standard training on the model versus those undergoing motion training prior to attempting cannulation on the BCMT.

\section{Participant selection and group allocation}

Endoscopy trainees without prior experience in ERCP (including simulator training) but with experience in upper and lower $\mathrm{Gl}$ endoscopy (>100 cases each) from seven centers in Europe (Bucharest, Rome, Zagreb, Bologna, Oxford, Brussels, and Strasbourg) were invited to participate in the study. Trainees included in the study were randomized to undergo motion training followed by standard cannulation training (motion training group) or standard cannulation training alone (standard train-

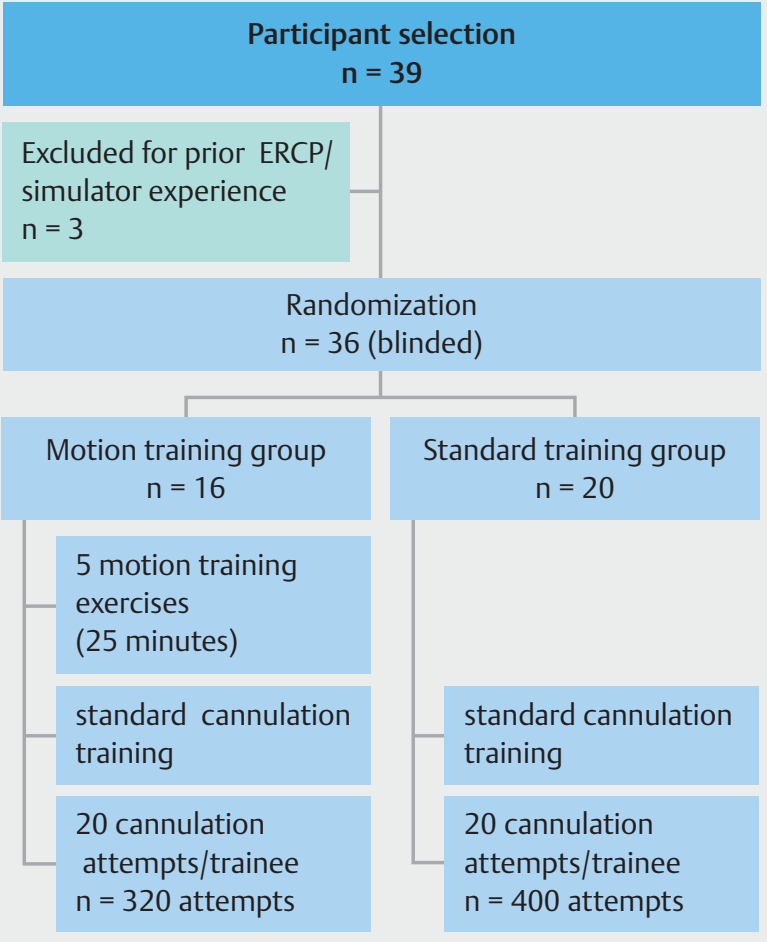

Fig. 1 Flowchart of the E-motion study (according to the CONSORT criteria).

ing group) on the mechanical simulator; both trainees and trainers were aware of the group allocation of the trainees during the study ( $\triangleright$ Fig. 1). Because this was an investigator-driven initiative that initially included only two centers, block randomization could not be implemented and sequentially numbered sealed opaque envelopes prepared at each individual center were used to allocate participants to their respective study group.

\section{Training protocol}

Cannulation training was performed using the Boškoski-Costamagna mechanical endoscopic simulator (Cook Medical, Limerick, Ireland) consisting of a lightweight metal cage and plastic components to represent the upper gastrointestinal tract. The simulator provides trainees with a suitable environment to learn and practice key maneuvers in ERCP training such as scope handling and correct positioning in front of the papilla, cannulation of the desired duct, stone extraction or stent placement [9]. Selective cannulation, one of the cornerstones of successful ERCP as well as one of the most complex parts of the procedure is simulated with the use of a non-biological replica of the papilla made out of latex with a transparent biliopancreatic duct system attached. There are several types of papillae available (small orifice, large orifice, common channel or parallel ducts for biliary and pancreatic access) [10] and adjustment of its position is possible for easier or more difficult access, with the intention of simulating known anatomical variants ( $\triangleright$ Fig.2). The model is ergonomic and quick to assemble 

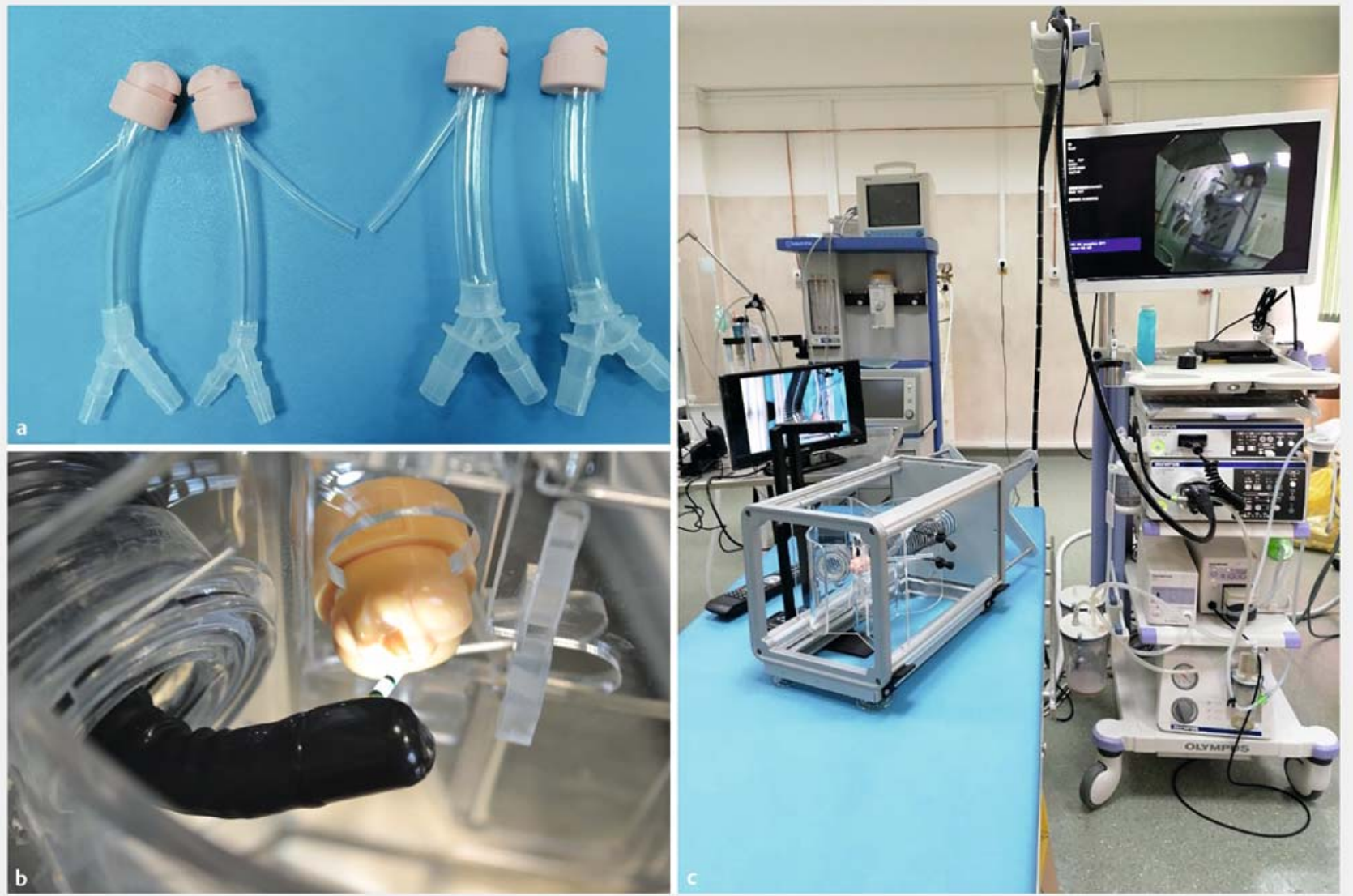

- Fig. 2 The Boškoski-Costamagna ERCP trainer (Cook Medical, Limerick, Ireland) a Non-biological cannulation models consisting of a papilla and biliopancreatic ducts in various anatomical designs (for easy, medium and difficult cannulation). b Cannulation attempt of the papilla using a standard duodenoscope and sphincterotome. c Complete simulator setup consisting of: endoscopy trolley, standard duodenoscope, the Boškoski-Costamagna ERCP trainer and simulated fluoroscopy unit with the aid of a HD camera and monitor

and it uses actual duodenoscopes and accessories, making it easy and convenient to use in variable teaching settings.

\section{Motion training}

Trainees randomized to the motion training group initially underwent motion training, which consisted of completing five tasks, such as writing and drawing, using the mechanical simulator, duodenoscope, and a specially modified $10 \mathrm{Fr}$ catheter with a graphite tip end before standard training and any attempt at cannulation of the papilla replica. For this purpose, the papilla on the model was replaced with a piece of white paper ( $\triangleright$ Fig.3) and the trainees were asked to try writing their names and draw simple shapes of their own choice. For each task, they were allotted 5-minute slots and they did not receive any verbal or hands-on instruction from their supervisors.

\section{Standardized cannulation training}

Both groups received standardized cannulation training using the model. This was performed at each center by an expert endoscopist (>2000 procedures), with the aim of explaining the practical aspects of selective common bile duct cannulation.
Briefly, the expert at each participating center explained the basic theory of scope handling with an emphasis on using a side-viewing scope, positioning in front of the papilla, and use of various dedicated accessories for ERCP. This theoretical briefing was followed by a demonstration of cannulation technique by the expert operator, using the mechanical simulator and highlighting the essential steps of cannulation: scope insertion, positioning in front of the papilla, use of the duodenoscope, wheels, shaft, and elevator, and selective cannulation using a sphincterotome and a 0.035 i guidewire ( $\bullet$ Video 1 ).

Following this demonstration, trainees proceeded to perform attempt cannulation on the mechanical model, under the supervision of the expert endoscopist.

\section{Cannulation attempts}

Trainees in both groups attempted deep cannulation of the common bile duct using a standard sphincterotome and a $0.035 \mathrm{i}$ guidewire with the local expert handling the accessories.

The trainees were required to complete a total of 20 cannulation attempts on four different papilla models, each attempt being timed from the moment the sphincterotome was out of the duodenoscope channel to the moment when deep cannula- 

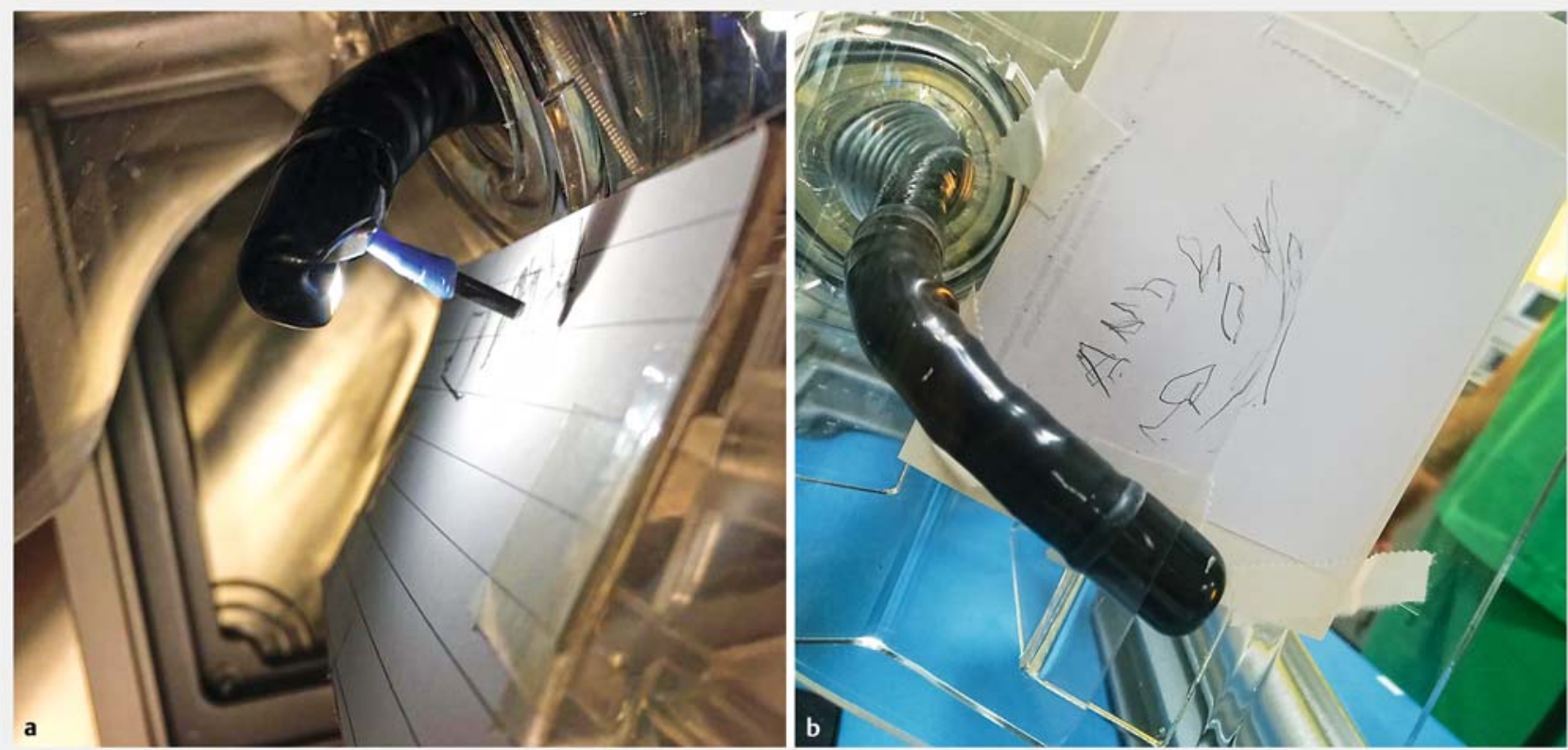

- Fig. 3 Motion training. Attempt at writing using a standard duodenoscope with a modified catheter with a graphite tip on a white piece of paper.

- Table1 Papilla types used for cannulation attempts.

\begin{tabular}{|l|l|l|l|}
\hline Attempts $\mathbf{0 - 5}$ & Attempts 6-10 & Attempts 11-15 & Attempts 16-20 \\
\hline $\begin{array}{l}\text { Common bilio-pancreatic channel, } \\
\text { normal inclination }\end{array}$ & $\begin{array}{l}\text { Common bilio-pancreatic channel, } \\
\text { difficult inclination }\end{array}$ & $\begin{array}{l}\text { Separate bile duct channel, } \\
\text { normal inclination }\end{array}$ & $\begin{array}{l}\text { Separate bile duct channel, } \\
\text { difficult duct anatomy }\end{array}$ \\
\hline
\end{tabular}

tion of the common bile duct was confirmed by direct visualization of the guidewire inside the transparent duct. If cannulation was not achieved after 5 minutes, the attempt was considered a failure. Four different difficulty levels of cannulation were simulated by adjusting the papilla type and angulation ( $\vee$ Table 1 and $>$ Fig. 2a)

Each cannulation was graded by the supervisor using a 4point scoring system taken from The endoscopic ultrasound (EUS) and ERCP Skills Assessment Tool (TEESAT). This validated scale uses a 4-point scoring system: 1 (superior), achieves task independently; 2 (advanced), achieves task with minimal verbal instruction; 3 (intermediate), achieves task with multiple verbal instructions; and 4 (novice), unable to complete the procedure, and was previously validated as an assessment tool for ERCP trainees [11]. For each timed cannulation, trainees were allowed 1 minute of unassisted attempts, following which, if deep cannulation of the desired duct was not achieved, verbal instructions were offered to assist with cannulation.

\section{Outcome measures}

The main outcome measure was time to successful selective cannulation of the common bile duct on the mechanical model. Trainee performance while cannulating, as assessed by the TEESAT score, was the secondary outcome measure of the study.

\section{Sample size estimation}

Based on data from the validation study of the Boškoski-Costamagna model [8] we assumed a mean cannulation time for the novice endoscopists of 120 seconds. We calculated that 170 procedures would be required to detect a $25 \%$ decrease in

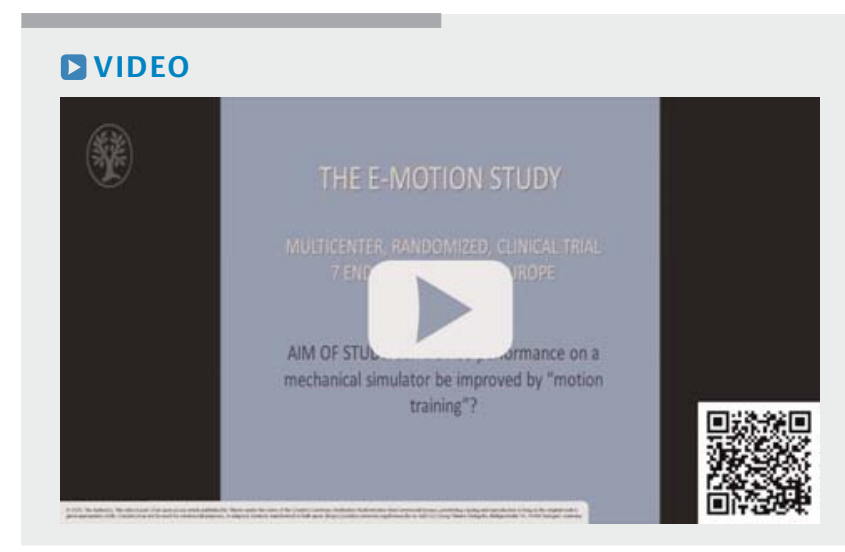

Video 1 The Emotion Study: Motion training, theoretical briefing and cannulation attempts performed on the BoskoskiCostamagna ERCP trainer. 
- Table 2 Potential risk factors for delayed cannulation.

\begin{tabular}{|c|c|c|}
\hline Factor & Standardized Coefficient $(95 \% \mathrm{Cl})$ & $P$ value \\
\hline Study group (E-motion/standard) & $0.077(0.513-19.515)$ & $0.039^{1}$ \\
\hline Type of papilla (1-4) & $0.077(0.235-8.678)$ & $0.039^{1}$ \\
\hline Training center & $0.059(-0.521-4.917)$ & 0.113 \\
\hline
\end{tabular}

Table 3 Comparison of cannulation times between the two study groups according to the type of papilla.

\begin{tabular}{|c|c|c|c|c|c|c|c|c|}
\hline \multirow{2}{*}{$\begin{array}{l}\text { Papilla type } \\
\text { Group }\end{array}$} & \multicolumn{2}{|l|}{1} & \multicolumn{2}{|l|}{2} & \multicolumn{2}{|l|}{3} & \multicolumn{2}{|l|}{4} \\
\hline & MT & ST & MT & ST & MT & ST & MT & ST \\
\hline Median cannulation time (s) & 31.5 & 42 & 33.5 & 47 & 38.5 & 51 & 41 & 48 \\
\hline Minimum(s) & 3 & 9 & 3 & 6 & 12 & 5 & 5 & 12 \\
\hline Maximum(s) & 300 & 300 & 300 & 300 & 300 & 300 & 300 & 300 \\
\hline$P$ value $^{1}$ & \multicolumn{2}{|l|}{$0.002^{2}$} & \multicolumn{2}{|l|}{0.094} & \multicolumn{2}{|l|}{0.298} & \multicolumn{2}{|c|}{0.052} \\
\hline $\begin{array}{l}\text { MT, motion training group; ST, standard training group. } \\
{ }^{1} \text { Mann-Whitney U test. } \\
{ }^{2} \text { Statistically significant using the Mann Whitney U test. }\end{array}$ & & & & & & & & \\
\hline
\end{tabular}

mean cannulation times with $90 \%$ power at a 0.05 significance level. However, taking into account the fact that each trainee would attempt multiple procedures, we adjusted the sample size to account for the inflation effect [4] and assumed that a minimum of 16 trainees in each group (32 in total) should perform 20 attempts per trainee to achieve adequate statistical power as well as a clinically relevant study design, in accordance with previous studies of ERCP training [4].

\section{Data management and statistical analysis}

All participating centers collected data prospectively using a standard report form (Appendix 1), completed by the trainer after each attempt at cannulation. Data were collected in a centralized database and analyzed using SPSS v. 22.

Bivariable analysis between the two study groups was carried out using the Mann Whitney $U$ and Kruskall-Wallis tests for comparing non-parametric variables and the chi-square test for dichotomic variables. Multivariate analysis using logistic and linear regression was carried out to account for the potential confounders arising from differences in training methods between the various participating centers. $P$ values were considered statistically significant if $<0.05$.

\section{Ethical concerns}

The study did not include any patients and all cannulation attempts were made using a mechanical simulator; approval from the local ethical committee was obtained by each participating center prior to study inception in accordance with the Declaration of Helsinki. Both trainees and local experts signed an informed consent regarding the collection and storage of personal data.

\section{Results}

Thirty-six trainees without prior ERCP experience from seven ERCP training centers were included in the study (Appendix 2). Sixteen trainees (44.4\%) were allocated to the motion training group and 20 (55.6\%) were allocated to the standard training group; they completed a total of 720 supervised attempts of selective cannulation of the common bile duct on the BCMT.

Successful cannulation of the bile duct was achieved in 698 of 720 attempts (96.9\%), with no significant difference between the two study groups ( $96.5 \%$ motion training group vs. $97.25 \%$ Standard cannulation group, $P=0.66$ ). Multivariable analysis using logistic regression showed that there was a statistically significant difference between centers in terms of successful cannulation, independent of study group and papilla type, with trainees from one individual center performing significantly better in terms in successful cannulation (OR 11.7, $95 \% \mathrm{Cl} 1.2-107.6)$.

Trainees in the motion training group had significantly lower median cannulation times compared to trainees in the standard group ( 36 vs. 48 seconds, minimum cannulation time of 3 and 4 seconds respectively, maximum cannulation time of $300 \mathrm{sec}$ onds for both groups; $P<0.001)$. This finding was confirmed in multivariable analysis using linear regression, where group allocation and type of papilla were identified as independent risk factors for prolonged cannulation time ( $\downarrow$ Table 2 ).

On subgroup analysis ( $\triangleright$ Table 3 ), this difference was only statistically significant for the first papilla configuration (median times 31 s vs. 42 s, $P=0.002$ ), with further timed cannulation attempts not differing significantly between the two study groups. 
Overall, time to cannulation increased as the level of complexity increased, with median times of 36 seconds, 41.5 seconds, 46.5 seconds and 45 seconds for types 1 to 4 , respectively, across the entire study population $(P=0.005)$.

Trainees in the motion training group had better technical performance than the standard training group, according to the supervisors' assessment, achieving TEESAT scores of 1 or 2 in 255 of 320 attempts (79.7\%) compared to 284 of 400 (71\%) in the standard training group ( $P=0.009$ chi-square). Multivariable analysis using logistic regression confirmed this finding, with standard group allocation being a risk factor for achieving less satisfactory TEESAT scores (OR 1.86, $95 \%$ Cl 1.29-2.72) after accounting for potential confounders such as papilla type and training center.

\section{Discussion}

The main focus of our study was to assess whether an innovative, out-of-the-box but intuitive method of training on a mechanical simulator can help improve a trainee's basic understanding of the procedure and speed up the learning curve for such complex technical skills as selective cannulation of the bile duct.

Our main finding was that trainees receiving prior motion training performed better than the standard training group, both in terms of cannulation times and overall performance as assessed by their supervisors. This effect was, however, restricted to the early part of the training, suggesting a plateau effect whereby the standard training group could catch up to the motion training group after the first cannulation attempts. This plateau effect has also been reported by some other trials focused on simulator training in endoscopy, particularly in the field of colonoscopy $[12,13]$.

Traditional training following the "master-apprentice" method where trainees learn by trial and error under the supervision of an expert endoscopist is, in most centers, still the only teaching method available. Despite the trainees having experience in forward-viewing endoscopy, such skills may prove to be insufficient when handling the side-viewing duodenoscope and associated accessories, which puts the patient at risk while resulting in longer operating room time [14] and higher costs.

Although it has gained popularity, simulator training in advanced endoscopy is yet to be included in training programs, because of the lack of published experience to indicate its impact on clinical outcome. However, there is evidence that simulator training in ERCP improves basic skills and translates into higher successful cannulation rates $[3,4]$ and faster cannulation time [4] with overall higher performance scores [3].

Different simulators are available to aid ERCP training, such as mechanical and computer-based simulators and animal models. Mechanical simulators are relatively cheap and quick to install, without requiring special preparation or facilities, and display realism to some extent, despite not offering the same tactile sensation as animal models [15]. However, mechanical simulators provide the opportunity of unlimited repetition of specific tasks while adjusting difficulty levels to suit more advanced trainees and they also allow the use of real scopes and accessories. Because of their versatility, mechanical simulators have outperformed both computer-based and exvivo models with respect to trainee preference and basic skill development across several studies [16-18].

New innovative teaching methods are gradually being introduced to simulation-based training. In a recent study, Perretta et al. showed the feasibility of modifying a laparoscopy trainer to allow flexible endoscopy training on this model. Their model adopted some basic tasks derived from laparoscopy training to simulate endoscopic procedures such as snaring, injection, clipping and even selective cannulation [19].

To our knowledge, our trial is the first to explore alternative ways of using mechanical simulators with the aim of improving trainee motor skills in the early phases of training in ERCP. The concept of using unfamiliar instruments (such as an endoscope and a simulator) to conduct more familiar tasks is also the focus of research in virtual reality simulators in endoscopy, but there are currently no long-term data about the impact of such training in real-life practice.

Our study has several strengths underscoring the validity of the results. First, this was a large, randomized, multicenter trial that recruited trainees and trainers from very different practice backgrounds, which supports the generalizability of our findings. Furthermore, our study used a validated model that was independently shown to have good face and construct validity [8], as well as a validated tool to assess trainee performance, the TEESAT score. Inasmuch as ERCP training, and selective cannulation of the bile duct in particular, can be standardized, our study achieved a controlled environment where trainees could be objectively assessed.

Several limitations inherent in our study design need to be taken into account when interpreting the results. First, the supervisors were not blinded to the group allocation of the trainees, which could potentially induce a bias in evaluating trainee performance. However, we believe the potential bias of this limitation is mitigated by the fact that the main outcome measure was represented by the cannulation time, which is not subject to evaluation bias. Furthermore, the differences found between the motion training group and the standard group were consistent for both the primary and secondary outcome measure, which suggests the risk of bias is very low. Second, the study design meant that trainees in the motion training group were allowed more time on the model (up to 25 minutes in total) compared to the standard group, which could account for the early advantage in performance on the model. However, there is no way to mitigate this bias other than looking at longterm outcomes of the trainees, which was outside the scope and possibilities of this study. Third, although statistically significant, it is unclear whether the difference in median cannulation time between the two study groups could be clinically significant when trainees start their hands-on training. This aspect is particularly important because the median cannulation times on our model were quite short in both study groups (below $60 \mathrm{~s}$ in both groups), significantly shorter than those reported by van der Wiel at al. in their trial on the BCMT [8] which was the basis for our study design. While we acknowledge the difference is very unlikely to have any clinical impact on its own, and 
while also underscoring the fact that faster might not actually translate into better cannulation skills in real-life practice, we believe it to represent a surrogate marker for better skill acquisition in the early phases of training, warranting further exploration of the utility of this type of simulator-based training. Another potential limitation stems from the design of our study, which lacked block randomization and relied on a simple randomization process at each study site, resulting in an unbalanced distribution of trainees in the two study groups (16 vs 20). We aimed to address the potential bias of this aspect by conducting multivariable regression analysis for each of the outcome variables to minimize the risk of bias and the results of this analysis were consistent with the findings of our univariate analysis. Consequently, despite the aforementioned limitations, we believe that the findings of our investigator-driven study are encouraging and warrant further investigation to assess how this innovative training method could further be refined and incorporated into everyday practice.

\section{Conclusion}

In conclusion, we have shown that motion training on the Boškoski-Costamagna mechanical trainer leads to swifter cannulation times and better overall performance in first-time trainees in ERCP techniques. Future studies should assess the long-term impact of such innovative training methods by looking at trainee performance during the hands-on training period and beyond, using clearly defined outcomes such as cannulation rates, technical success rates, and validated assessment scores such as the TEESAT score.

\section{Acknowledgments}

The authors would like to acknowledge the following members of the E-Motion Study Group who participated in the training sessions for the study: Ben Souda J., Benjelloun N., Benotmane S., Bibbo S., Burrelli Scotti G., Buttitta F., Carlini E., Chiricuta A., Cirota G., De Siena M., Di Maurizio L., Ejaz T., Estévez Schwarz L., Gibiino G., Hussenbux A., Jelakovic M., Kocev M., Kundiona I., Laterza L., Lopetuso L., Marmo C., Moirana E., Napoli M., Ouakam J., Papparella L., Popescu D., Rizzatti G., Sathananthan D., Seelinger B., Semprucci G., Souiki T., Tamanini G., Thayalasekaran S., Webb G., and Zacheu H.

\section{Competing interests}

Dr. Costamagna is a consultant for Cook Medical, Boston Scientific and Olympus. Dr. Boskoski is a consultant for Cook Medical, Boston Scientific and Apollo Endosurgery, a holder of a research grant from Apollo Endosurgery and a Scientific Board Member for Endo Tools.

\section{References}

[1] Wani S, Hall M, Wang AY et al. Variation in learning curves and competence for ERCP among advanced endoscopy trainees by using Cumulative sum analysis. Gastrointest Endosc 2016; 83: 711-719 e11

[2] Voiosu T, Balanescu P, Voiosu A et al. Measuring trainee competence in performing endoscopic retrograde cholangiopancreatography: A systematic review of the literature. United European Gastroenterol ] 2019; 7: 239-249

[3] Leung J, Liao W, Wong S et al. A RCT of mechanical simulator practice and usual training vs. usual training on novice trainee clinical ERCP performance. Gastrointest Endosc 2009; 69: AB141

[4] Lim B, Leung J, Yen D et al. Effect of ERCP mechanical simulator (EMS) practice on trainees? ERCP performance in the early learning period: U.S. multi-center randomized controlled trial. Am J Gastroenterol 2011; 106: 300-306

[5] Gregoire D, Rateb G. Learning ERCP with the Boskoski-Costamagna mechanical simulator: a single trainee's experience. Endoscopy 2018; 50: $\$ 163$

[6] van der Wiel SE, Kuttner Magalhaes R, Rocha Goncalves CR et al. Simulator training in gastrointestinal endoscopy - From basic training to advanced endoscopic procedures. Best Pract Res Clin Gastroenterol 2016; 30: 375-387

[7] Ekkelenkamp VE, Koch AD, de Man RA et al. Training and competence assessment in Gl endoscopy: a systematic review. Gut 2016; 65: 607615

[8] van der Wiel S, Koch A, Bruno M. Face and construct validity of a novel mechanical ERCP simulator. Endosc Int Open 2018; 6: E758-E765

[9] Boskoski I, Landi R, Bove V et al. New methods of teaching axis in ERCP: can I write my name? VideoGIE 2018; 03: 45-46

[10] Boškoski I, Costamagna G. The Boškoski-Costamagna ERCP Trainer: from dream to reality. Endoscopy 2016; 48: 593

[11] Wani S, Keswani RN, Petersen B et al. Training in EUS and ERCP: standardizing methods to assess competence. Gastrointest Endosc 2018; 87: $1371-1382$

[12] Eversbusch A, Grantcharov TP. Learning curves and impact of psychomotor training on performance in simulated colonoscopy: a randomized trial using a virtual reality endoscopy trainer. Surg Endosc 2008; 18: 1514-1518

[13] Jirapinyo P, Abidi WM, Aihara $\mathrm{H}$ et al. Preclinical endoscopic training using a part-task simulator: learning curve assessment and determination of threshold score for advancement to clinical endoscopy. Surg Endosc 2017; 31: 4010-4015

[14] Voiosu T, Voiosu A, Bengus A et al. Trainee involvement increases precut rates and delays access to the common bile duct without an increase in procedure-related adverse events: a brave new world of ERCP training? Romanian J Int Med 2017; 56: 55-61

[15] Sedlack R, Petersen B, Binmoeller K et al. A direct comparison of ERCP teaching models. Gastrointest Endosc 2003; 57: 886-890

[16] Leung JW, Yen D. ERCP training - the potential role of simulation practice. J Interv Gastroenterol 2011; 1: 14-18

[17] Leung J, Lim B, Ngo C et al. Head-to-head comparison of practice with endoscopic retrograde cholangiopancreatography computer and mechanical simulators by experienced endoscopists and trainees. Dig Endosc 2012; 24: 175-181

[18] Leung JW, Wang D, Hu B et al. A head-to-head hands-on comparison of ERCP mechanical simulator (EMS) and ex-vivo porcine stomach model (PSM). J Interv Gastroenterol 2011; 1: 108-113

[19] Habaz I, Perretta S, Okrainec A et al. Adaptation of the fundamentals of laparoscopic surgery box for endoscopic simulation: performance evaluation of the first 100 participants. Surg Endosc 2019; 33: 34443450 